\title{
THERMONUCLEAR RUNAWAY MODELS FOR SYMBIOTIC NOVAE
}

\author{
Scott J. Kenyon \\ Smithsonian Astrophysical Observatory \\ Harvard-Smithsonian Center for Astrophysics \\ 60 Garden Street \\ Cambridge, MA 02138 USA
}

\begin{abstract}
This paper reviews the basic physics of thermonuclear runaways on the surfaces of accreting white dwarf stars, with a special emphasis on understanding the evolution of symbiotic novae.
\end{abstract}

\section{Introduction}

The eruptions of the small class of objects known as symbiotic novae are very different from those experienced by classical symbiotic binaries such as Z And and CI Cyg. As reviewed by Viotti in this volume (see also Kenyon 1986, Chapter 5), symbiotic nova eruptions are characterized by a slow rise to visual maximum ( $\sim$ a few years) followed by a very tedious decline ( - many decades). Observations suggest the bolometric luminosity, $\mathrm{L}_{\mathrm{bol}}$, of a symbiotic nova remains roughly constant following visual maximum, although the visual luminosity, $\mathrm{L}_{\mathrm{vis}}$, decreases by a factor of $\sim 100$. This evolution of $\mathrm{L}_{\mathrm{bol}}$ and $\mathrm{L}_{\mathrm{vis}}$ with time is very similar to that observed in classical novae (see Gallagher and Starrfield 1978), which suggests a common eruption mechanism for these two types of novae.

It is well-established that enuptions of classical novae result from thermonuclear runaways on the surfaces of white dwarf stars. The basic physical model consists of a short period binary system ( $\mathrm{P}_{\text {orb }} \sim$ hours), in which a lobe-filling red dwarf transfers material into an accretion disk surrounding a white dwarf. A hydrogen-rich atmosphere builds up on the white dwarf's surface, and eventually the pressure at the base of this envelope is sufficient to ignite the accreted material. Since the white dwarf atmosphere is degenerate, the ignition is explosive and material is accelerated outward. The white dwarf increases in luminosity by 15 mag to $M_{V} \sim-7$ during the eruption, and the radius of the nova photosphere expands to envelop the entire binary system (Fujimoto 1982a,b; MacDonald 1983). Nuclear reactions and gravitational stirring of the nova photosphere by the red dwarf generate enough energy to expel a large fraction of the accreted envelope from the binary system at velocities of 300 to $2000 \mathrm{~km} \mathrm{~s}^{-1}$ (MacDonald 1980). Once the accreted material has been either burned or ejected, the nova fades to relative insignificance over a period of several months.

The major physical difference between a symbiotic nova and a classical nova is that the mass-losing star in a symbiotic binary is a red giant rather than a red dwarf. Observations indicate that symbiotic novae are detached binaries, so mass transfer occurs via a wind instcad of tidal overflow. The large orbital periods of symbiotic novae, $P_{\text {orb }} \geq 2-3 \mathrm{yr}$, also guarantee that the expanding white dwarf photosphere lies well within its inner Lagrangian surface throughout the eruption, and thus these systems do not become common envelope 
binaries at visual maximum. This fact may explain why symbiotic novae decline on a time scale which is more comparable to the nuclear time scale, as opposed to the classical novae, in which gravitational stirring of the nova photosphere by the red dwarf companion appears to cause a more rapid decline than can be accounted for by the combined actions of nuclear burning and mass ejection in a normal stellar wind. Thus, the symbiotic novae may provide a better laboratory in which to study thermonuclear runaways than classical novae.

\section{Basic Physics}

The outburst cycle of a symbiotic nova is conveniently divided into two phases, a quiescent or "off" phase and an eruptive or "on" phase. The white dwarf patiently accretes matter from its companion during quiescence, and its physical appearance to an outside observer depends on the mass and radius of the white dwarf, $M_{w d}$ and $R_{w d}$, and the mass accretion rate, $\dot{M}$. Material settles onto the white dwarf fairly smoothly if $\dot{M}$ is fairly small (say, $\leq 10^{-10} \mathrm{M}_{\odot} \mathrm{yr}^{-1}$ ), while a hydrogen buming shell is created near the white dwarf's surface if the accretion rate is large. Paczynski and Zytkow (1978) demonstrated that there is a range in accretion rates such that material bums completely as it is accreted, and therefore does not accumulate on the white dwarf's surface. This steady burning limit was investigated in detail by Iben (1982), who found a minimum accretion rate of:

$$
\dot{\mathrm{M}}_{\text {steady,min }} \approx 1.32 \times 10^{-7} \mathrm{M}_{\odot} \mathrm{yr}^{-1}\left[\frac{\mathrm{M}_{\mathrm{wd}}}{1 \mathrm{M}_{\odot}}\right]^{3.57} \text {. }
$$

Since the material bums as it is accreted, this limit results in a white dwarf luminosity of

$$
\mathrm{L}_{\text {steady,min }} \approx 10^{4} \mathrm{~L}_{\odot}\left[\frac{\mathrm{M}_{\mathrm{wd}}}{1 \mathrm{M}_{\odot}}\right]^{3.57} .
$$

The maximum luminosity that can be achieved by a steadily burning white dwarf is given by the Paczynski (1970) - Uus (1970) core mass-luminosity relationship

$$
\mathrm{L}_{\text {plateau }} \approx 59,250 \mathrm{~L}_{\odot}\left(\mathrm{M}_{\mathrm{wd}}-0.52 \mathrm{M}_{\odot}\right) \text {, }
$$

which results in a maximum steady accretion rate of:

$$
\dot{\mathrm{M}}_{\text {steady,max }} \approx 6 \times 10^{-7} \mathrm{M}_{\odot} \mathrm{yr}^{-1} \mathrm{X}^{-1}\left(\mathrm{M}_{\mathrm{wd}}-0.52 \mathrm{M}_{\odot}\right) \text {, }
$$

where $\mathrm{X}$ is the mass fraction of hydrogen in the accreted envelope.

If the accretion rate exceeds the maximum steady rate $\left(\dot{M}>\dot{M}_{\text {steady,max }}\right)$, then the additional (unburnt) material expands into an extended atmosphere similar to that of a red giant. On the other hand, if the accretion rate is less than the minimum steady rate $\left(\dot{\mathrm{M}}<\dot{\mathrm{M}}_{\text {steady,min }}\right)$, then the radius of the stellar photosphere remains comparable to the white dwarf's radius (Iben 1982). Explosive shell flashes occur in both of these configurations. The helium burning shells of red giants are thermally unstable, resulting in recurrent helium shell flashes which will not be discussed here (Iben and Renzini 1983). When $\dot{M}<$ $\dot{\mathrm{M}}_{\text {steady,min }}$, both the hydrogen and helium shells are thermally unstable; the hydrogen shell flashes result in objects that are observed as classical novae and symbiotic novae, while the less frequent helium flashes may give rise to supernova eruptions (Iben and Tutukov 1983).

Various numerical and semi-analytical calculations have investigated the time evolution of accreting white dwarfs when $\dot{M}<M_{\text {steady,max }}$ (Paczynski and Żytkow 1978; Sion, Aciemo, and Tomczyk 1979; Kutter and Sparks 1980; Fujimoto 1982a,b; MacDonald 1980, 1983; Prialnik, et al. 1982; Nariai, Nomoto, and Sugimoto 1980; Starrfield, Sparks, and Truran 
1984; Sion and Starrficld 1985; Prialnik 1986). Fujimoto and MacDonald have shown that hydrogen buming commences when the pressure at the base of the accreted envelope reaches a critical value. The material does not expand significantly during the early stages of shell burning, because the atmosphere is partially (in some cases, completely) degenerate. The temperature at the base of the accreted layer increases exponentially until the degeneracy is lifted and the atmosphere responds on a hydrodynamic time scale ( $\sim$ minutes). Because the energy released in hydrogen buming $\left(-10^{18} \mathrm{erg} \mathrm{g}^{-1}\right)$ exceeds the binding energy of the accreted envelope $\left(\sim 10^{19} \mathrm{erg}^{-1}\right)$, the shell flash can result in a rapid ejection of material if most of the hydrogen fuel can be processed via the CNO cycle before the atmosphere expands (and the resulting drop in the density and temperature of the burning shell quenches the runaway). Thus the most important parameter describing the evolution of the shell flash is the ratio of nuclear energy generated while the atmosphere is degenerate, $E_{\text {nuc }}$, to the envelope's binding energy, $E_{\text {bind. }}$. Violent runaways occur if $E_{n u d} / E_{\text {bind }} \geq 1$, and detailed numerical calculations yield expansion velocities of $1000-3000 \mathrm{~km} \mathrm{~s}^{-1}$. Weak shell flashes are predicted if $\mathrm{E}_{\text {nud }} / \mathrm{E}_{\text {bind }} \ll 1$, and such objects may not eject material dynamically.

The critical ratio, $\mathrm{E}_{\text {nud }} / \mathrm{E}_{\text {bind }}$, depends on several physical parameters: the mass and luminosity of the accreting white dwarf $\left(\mathrm{M}_{w d}\right.$ and $\left.L_{w d}\right)$, the mass accretion rate $(\dot{M})$, and the mass fraction of $\mathrm{CNO}$ nuclei in the accreted envelope $\left(\mathrm{Z}_{\mathrm{CNO}}\right)$. Since $\mathrm{E}_{\text {nuc }} / \mathrm{E}_{\text {bind }}$ increases if the accreted envelope is more degenerate, low white dwarf luminosities and low accretion luminosities favor violent eruptions. For a given $L_{w d}$ and $\dot{M}$, stronger eruptions can be achieved on a more massive white dwarf, because (i) a lower mass envelope is required to initiate the runaway (thereby decreasing $E_{\text {bind }}$ ) and (ii) the pressure at the base of the accreted envelope is larger for a given $M_{e n v}$ (thereby increasing $E_{n u c}$ ).

The relationship between $\mathrm{E}_{\text {nud }} / \mathrm{E}_{\text {bind }}$ and the $\mathrm{CNO}$ abundance depends on the nuclear physics involved with the main energy source for the runaway, the CNO cycle. For the temperatures that usually are reached in the hydrogen buming shell, $\mathrm{T}_{\text {shell }}-10^{8} \mathrm{~K}$, stable $\mathrm{C}$ and $\mathrm{N}$ nuclei fuse into the $\beta^{+}$unstable nuclei ${ }^{14} \mathrm{O}$ and ${ }^{15} \mathrm{O}$. The runaway must then "wait" for these nuclei to decay before energy production can continue. However, the lifetimes of these nuclei $(\sim 180 \mathrm{sec})$ are longer than the local hydrodynamic time scale, so the atmosphere expands before the nuclei decay. Thus, $\mathrm{E}_{\text {nuc }} / \mathrm{E}_{\text {bind }}$ is limited by the amount of energy which can be produced from the capture of 1-2 protons on every CNO nucleus:

$$
\left[\frac{E_{\text {nuc }}}{E_{\text {bind }}}\right] \sim 0.01\left[\frac{Z_{\text {CNO }}}{Z_{\mathrm{CNO}, \odot}}\right] \text {, }
$$

where $\mathrm{Z}_{\mathrm{CNO}, \odot}$ is the solar CNO abundance. It is apparent that a hydrodynamic runaway requires $\mathbf{Z}_{\mathrm{CNO}} \rightarrow \mathbf{Z}_{\mathrm{CNO}, \odot}$. Detailed numerical calculations have confirmed this basic point in detail: hydrodynamic or "fast" novae result if $\mathrm{Z}_{\mathrm{CNO}} \gg \mathrm{Z}_{\mathrm{CNO}, \odot}$ while hydrostatic or "slow" novae occur if $\mathbf{Z}_{\mathrm{CNO}}-\mathrm{Z}_{\mathrm{CNO}, \odot}$.

Important observational parameters characterizing shell flashes are the the time spent at quiescence, $\Delta \mathrm{t}_{\text {off }}$, and the time spent in eruption, $\Delta \mathrm{t}_{\mathrm{on}}$. If the mass of the accreted envelope needed for the runaway is $M_{\text {env }}$, the quiescent time scale can be estimated as:

$$
\Delta \mathrm{t}_{\text {off }} \approx 10^{4} \mathrm{yr}\left(\frac{\mathrm{M}_{\mathrm{env}}}{10^{-5} \mathrm{M}_{\odot}}\right)\left[\frac{10^{-9} \mathrm{M}_{\odot} \mathrm{yr}^{-1}}{\dot{\mathrm{M}}}\right]
$$

An upper limit to the time spent in the "on" state is the ratio of available nuclear energy, $\mathrm{E}_{\text {nuc }}$, to the luminosity at maximum, $\mathrm{L}_{\text {plateau: }}$

$$
\Delta \mathrm{t}_{\text {on }} \sim 40 \mathrm{yr}\left[\frac{\mathrm{M}_{\mathrm{env}}}{10^{-5} \mathrm{M}_{\odot}}\right]\left[\frac{2 \times 10^{4} \mathrm{~L}_{\odot}}{\mathrm{L}_{\text {plateau }}}\right]
$$


Table 1 - Time scales for Accreting $1 \mathrm{M}_{\odot}$ White Dwarfs (Iben 1982)

\begin{tabular}{lrr}
\hline \hline Accretion Rate $\left(\mathrm{M}_{\odot} \mathrm{yr}^{-1}\right)$ & $\Delta \mathrm{t}_{\text {off }}(\mathrm{yr})$ & $\Delta \mathrm{t}_{\text {on }}(\mathrm{yr})$ \\
\hline $10^{-7}$ & 38 & 18 \\
$10^{-8}$ & 630 & 21 \\
$10^{-9}$ & 15000 & 49 \\
$10^{-10}$ & 330000 & 110 \\
\hline
\end{tabular}

Characteristic values for $\Delta \mathrm{t}_{\text {on }}$ and $\Delta \mathrm{t}_{\text {off }}$ are given by Iben (1982) for a $1 \mathrm{M}_{\odot}$ white dwarf, and are summarized in Table 1 . The ratio $\Delta \mathrm{t}_{\text {off }} / \Delta \mathrm{t}_{\text {on }} \gg 1$ as long as $\dot{M}$ does not greatly exceed a few $\times 10^{-8} \mathrm{M}_{\odot} \mathrm{yr}^{-1}$, implying that accreting white dwarfs undergoing thermonuclear runaways should spend the bulk of their time in a quiescent state. Rapid recurrence time scales are possible only at very high accretion rates; systems displaying fairly frequent outbursts are therefore expected to be very intense ultraviolet sources at quiescence.

\section{Evolution of Symbiotic Novae}

Applications of the basic theory of hydrogen shell flashes in the envelopes of accreting white dwarf stars to symbiotic novae have been described by Tutukov and Yungel'son (1976, 1982), Paczynski and Żytkow (1978), Paczyński and Rudak (1980), Iben (1982), Kenyon and Truran (1983), and Shustov and Tutukov (1985). Tutukov and Yungel'son (1976) noted that the high energy photons likely to be produced by a steady shell source could probably account for the blue continuum and high energy emission lines observed in most quiescent symbiotic systems, and suggested that slight increases in the mass accretion rate might result in an eruption (especially if $\dot{M}$ during the outburst is larger than $\dot{\mathbf{M}}_{\text {steady,max }}$ as outlined above). They later investigated this model in more detail (Tutukov and Yungel'son 1982), and derived constraints on the red giant mass loss rate, $\dot{\mathrm{M}}_{\text {giant }}$, and the binary separation, $\mathrm{A}$, with the basic assumption of steady burning. Their results, $2 \mathrm{AU}<\mathrm{A}<20 \mathrm{AU}$ and $10^{-7} \mathrm{M}_{\odot} \mathrm{yr}^{-1}<\dot{\mathrm{M}}_{\text {giant }}<10^{-5} \mathrm{M}_{\odot} \mathrm{yr}^{-1}$, are in reasonable agreement with the orbital periods (Garcia, this volume) and mass loss rates (Taylor, this volume) found for most symbiotic stars.

Paczynski and Rudak proposed that outbursts of symbiotic stars naturally divide into two categories, which they called type I (Z And, CI Cyg, AG Dra) and type II (V1016 Cyg, V1329 Cyg, HM Sge). They suggested that type I symbiotics possess accreting white dwarfs with stable hydrogen burning shells; outbursts then result from variations in the accretion rate as described by Tutukov and Yungel'son. Type II symbiotic systems contain white dwarfs accreting at rates below $\dot{\mathrm{M}}_{\text {steady,min }}$, and experience hydrogen shell flashes. Kenyon and Truran (1983) pointed out that this idea could be understood in terms of eruptions which occur in degenerate white dwarf atmospheres (some type II systems) and others which begin in non-degenerate envelopes (all type I systems and some type II objects). Thus, it is convenient to divide a discussion of the time behavior of symbiotic novae into the cvolution of degenerate eruptions and the evolution of non-degenerate eruptions. 


\section{A. Degenerate Flashes}

If the accretion rate onto a white dwarf is fairly low $\left(\dot{\mathrm{M}} \leq 10^{-10} \mathrm{M}_{\odot} \mathrm{yr}^{-1}\right.$ or so), then the material can cool efficiently before it settles into the hydrogen-rich atmosphere (see Figure 1). In this situation, the temperature of the white dwarf atmosphere depends only on its luminosity, $\mathrm{L}_{\mathrm{wd}}$, which will be assumed to be fairly small $\left(\mathrm{L}_{\mathrm{wd}} \leq 0.1 \mathrm{~L}_{\odot}\right)$. The white dwarf envelope is then completely degenerate when the runaway commences, which guarantees an explosive flash as noted above.

The rise to visual maximum during a degenerate shell flash is characterized by (i) a rapid increase in bolometric luminosity at nearly constant radius, followed by (ii) a slow expansion at constant bolometric luminosity into an A-F supergiant configuration (Figure 2). During phase (i) the visual brightness of the white dwarf remains fairly constant, although ionization of material expelled by the giant should produce optical emission lines. Once the eruptive star enters phase (ii), its visual brightness increases significantly and overwhelms that of its giant companion. The visual luminosity at maximum can be estimated from the core mass-luminosity relation of equation (3) to be $=4000 \mathrm{~L}_{\odot}$ for a typical $\left(0.6 \mathrm{M}_{\odot}\right)$ white dwarf, and can be compared to the $100-200 \mathrm{~L}_{\odot}$ expected of a normal giant star.

The spectroscopic evolution of a degenerate shell flash can be outlined using spectrum synthesis techniques described by Kenyon and Webbink (1984). I will assume that the binary initially consists of an M2 III star in combination with a low luminosity, $0.8 \mathrm{M}_{\odot}$ white dwarf $\left(\mathrm{L}_{\mathrm{wd}} \sim 10^{-2} \mathrm{~L}_{\odot}\right)$ accreting material at a rate, $\dot{\mathrm{M}} \sim 10^{-10} \mathrm{M}_{\odot} \mathrm{yr}^{-1}$. The optical/ultraviolet spectrum predicted for this configuration appears in the lower left panel of Figure 3.

The rise to visual maximum begins with an increase in effective temperature at nearly constant radius (phase (i)). The maximum effective temperature for a $1 \mathrm{M}_{\odot}$ object is $\mathrm{T}_{\text {hot }}$ $2 \times 10^{5} \mathrm{~K}$, so the blackbody continuum of the rapidly evolving nova shifts from the UV into the X-ray spectral region. My models assume that all of the high energy photons from the hot object are locally absorbed by the red giant wind, so the nebular spectrum grows dramatically in strength as $\mathrm{T}_{\text {ho }}$ increases (Figure 3, upper left panel).

During the expansion phase (ii), the hot component evolves at constant luminosity from a very hot white dwarf into an A-F supergiant. The visual brightness of the system increases dramatically, and the emission lines become progressively weaker as $T_{\text {hot }}$ decreases. The spectroscopic evolution during this constant luminosity phase can be visualized by comparing the upper left panel of Figure $3\left(\mathrm{~T}_{\text {hot }}-2 \times 10^{5} \mathrm{~K}\right)$ with the upper right $\left(\mathrm{T}_{\mathrm{hot}}-7 \times 10^{4} \mathrm{~K}\right)$ and lower right $\left(\mathrm{T}_{\text {hot }} \sim 7 \times 10^{3} \mathrm{~K}\right)$ panels.

Following visual maximum, the hot component retraces its evolution in the H-R diagram. By analogy with classical novae, declining symbiotic novae should possess dense outflowing winds, and Wolf-Rayet features have been observed in several symbiotic novae (Kenyon 1986). Mass loss rates derived for these systems are typically $\sim 10^{-6} \mathrm{M}_{\odot} \mathrm{yr}^{-1}$, which is somewhat larger than the rate at which hydrogen is consumed in the burning shell $\left(\sim 5 \times 10^{-7} \mathrm{M}_{\odot} \mathrm{yr}^{-1}\right)$. Thus, the length of time spent in the "on" phase, $\Delta \mathrm{t}_{\text {on }}$, is apt to be somewhat shorter (perhaps a factor of -3 ) than estimated by equation (7).

\section{B. Non-Degenerate Flashes}

If the accretion rate onto the white dwarf exceeds $-10^{-9} \mathrm{M}_{\odot} \mathrm{yr}^{-1}$, then accreted material cannot cool before mixing with the white dwarf atmosphere. This heating of the white dwarf by newly arrived hydrogen-rich matter results in a non-degenerate envelope at the onset of the thermonuclear runaway, and causes a relatively weak shell flash. 


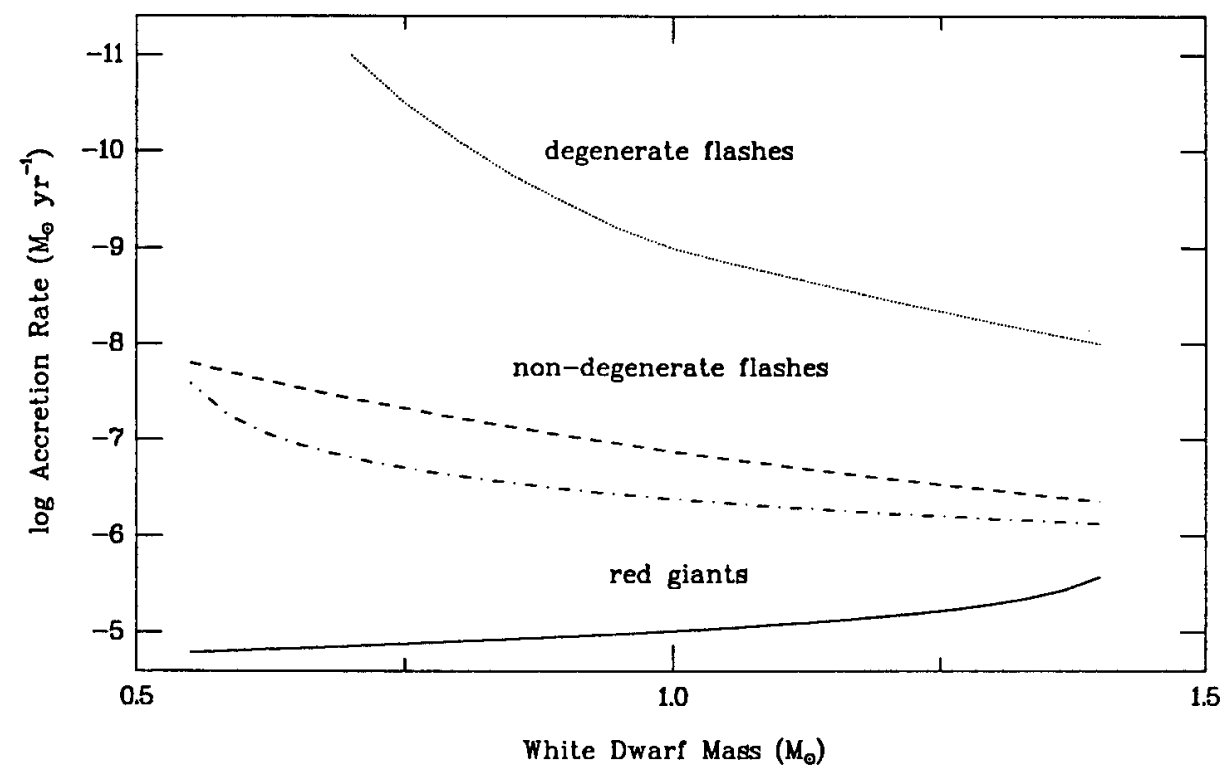

Figure 1 - Behavior of hydrogen shell flashes as a function of $\dot{M}$ and $M_{w d}$ for solar CNO abundance and $\mathrm{L}_{\mathrm{wd}} \leq \mathrm{L}_{\text {accr }} \leq 0.1 \mathrm{~L} \odot$. Steady-burning configurations are allowed between the dashed and dot-dashed lines. The Eddington limit has been plotted as the solid line at the bottom of the Figure.
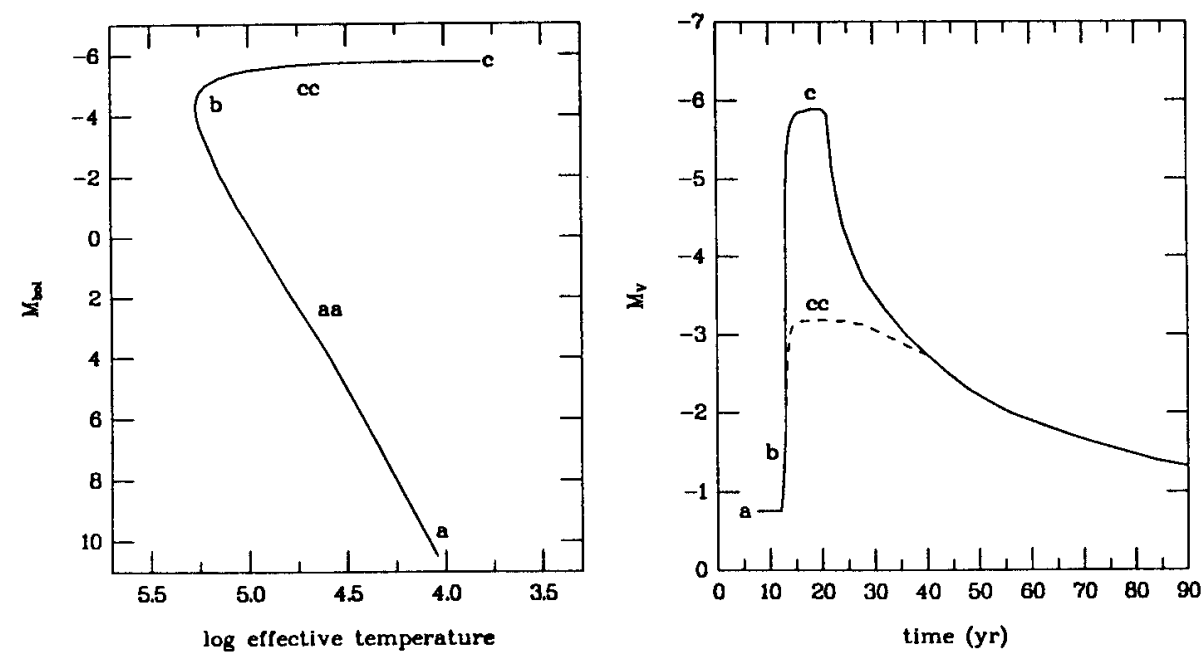

Figure 2 - Evolution of a thermonuclear runaway on a $0.8 \mathrm{M}_{\odot}$ white dwarf star. The left panel shows the behavior of the system in the H-R diagram, while the right panel displays theoretical light curves for degenerate ( $a-b-c)$ and non-degenerate (aa-b-cc) configurations. 

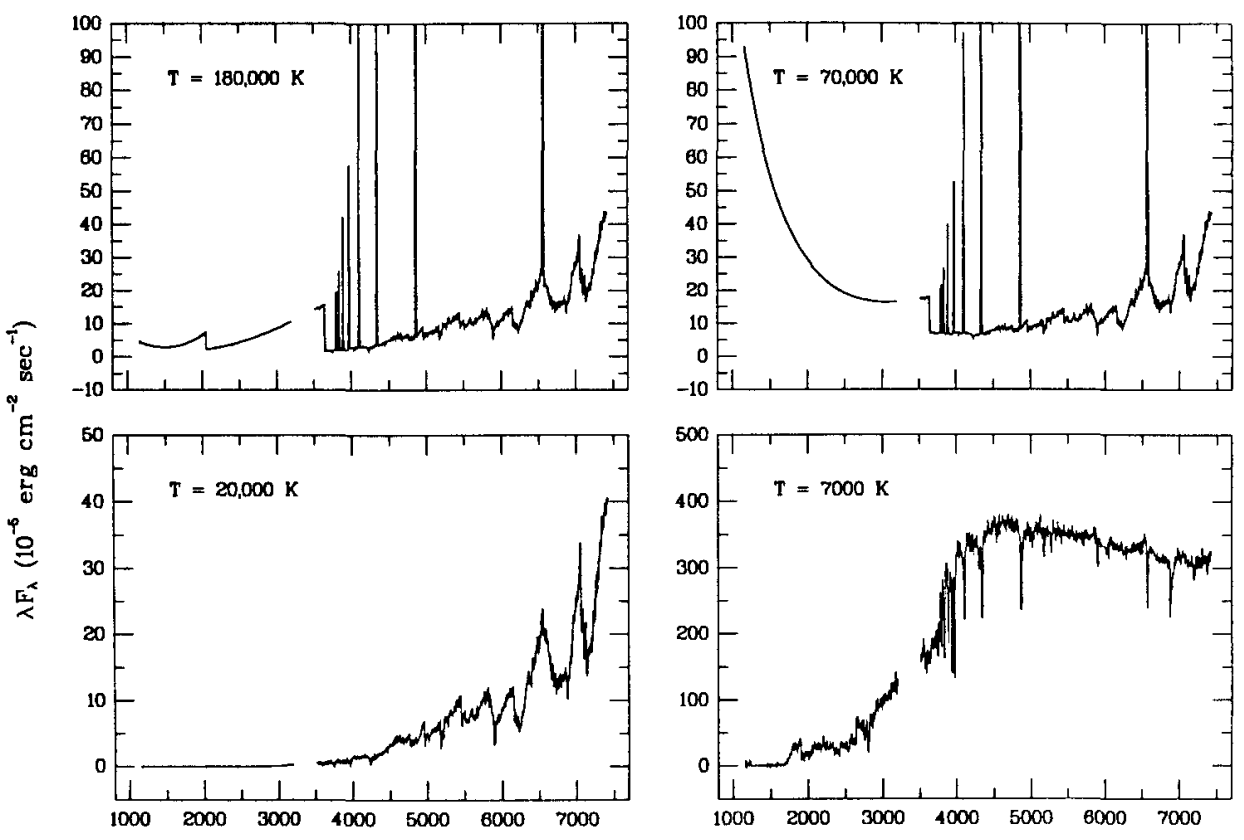

Wavelength $(\AA)$

Figure 3 - Synthetic spectra of a symbiotic nova as a function of effective temperature during the eruption. The models combine spectra of an M5 giant $(V=-1.5)$ with a hot stellar source at the indicated effective temperatures and an ionized nebula at a gas temperature of $10,000 \mathrm{~K}$. The plotted spectra correspond to marked points in the H-R diagram of Figure 2.

Unlike the degenerate shell flashes described above, weak non-degenerate flashes do not evolve into A-F supergiants and remain very hot throughout the cruption. Although these systems reach the plateau luminosity defined in equation (6), they make smaller excursions in the H-R diagram (e.g., aa-b-cc in Figure 2) and have a correspondingly smaller increase in visual brightness (Iben 1982; Kenyon and Truran 1983).

The spectroscopic evolution of a non-degenerate shell flash during the expansion phase (i) is essentially identical to that of a degenerate flash, although objects with comparatively weak eruptions may require a longer period of time to reach bolometric (and visual) maximum. The change in the optical/ultraviolet spectrum during phase (i) can be visualized by comparing the left panels of Figure 3 .

These systems spend a very short amount of time at temperatures, $T_{\text {hot }} \leq 10^{5} \mathrm{~K}$ in the constant luminosity phase (ii), and thus remain intense emission line sources throughout their eruptions. An example of a possible spectrum for such an object near visual maximum is shown in the upper right panel of Figure 3.

\section{The Symbiotic Novae}

The theory of hydrogen shell flashes predicts that accreting white dwarf stars will display two types of cruptive bchavior depending on the physical parameters of the white 
dwarf $\left(\mathrm{M}_{\mathrm{wd}}\right.$ and $\left.\mathrm{L}_{\mathrm{wd}}\right)$ and of the accreted material $\left(\dot{\mathrm{M}}\right.$ and $\left.\mathrm{Z}_{\mathrm{CNO}}\right)$. Degenerate flashes occur when the accretion luminosity $\left(\mathrm{L}_{\mathrm{accr}} \propto \mathrm{M}_{\mathrm{wd}} \dot{\mathrm{M}}\right)$ and the white dwarf luminosity are small $\left(\mathrm{L}_{\mathrm{wd}} \leq \mathrm{L}_{\mathrm{accr}} \leq 0.1 \mathrm{~L}_{\odot}\right)$ and the mass fraction of CNO nuclei is large $\left(\mathrm{Z}_{\mathrm{CNO}}>\mathrm{Z}_{\mathrm{CNO}, \odot}\right)$. White dwarfs undergo non-degenerate flashes when $L_{w d}$ or $L_{a c c r}$ is large $\left(\geq 1 L_{\odot}\right.$ ).

Detailed comparisons of the theory with observations suggest that both types of eruptions actually occur in symbiotic novae (Kenyon 1986; Viotti, this volume). It appears that AG Peg, RT Ser, RR Tel, and PU Vul have undergone degenerate flashes, and their evolution following visual maximum is consistent with the constant luminosity phase, $\mathrm{L}_{\text {bol }} \sim \mathrm{L}_{\text {plateau }}$, described above. Other symbiotic novae, V1016 Cyg, V1329 Cyg, and HM Sge, seem to have experienced non-degenerate flashes, as evidenced by their well-developed emission-line spectra at visual maximum.

Additional observations of these systems as they decline from visual maximum are important tests of the theory of hydrogen shell flashes. The newest symbiotic nova, PU Vul, is of special interest in this regard, because it has not yet evolved to high effective temperatures. Evolution of its spectrum, particularly in the X-ray and radio regions, should be most instructive.

\section{References}

Fujimoto, M.Y. 1982a. Astrophys. J., 257, 752.

Fujimoto, M.Y. 1982b. Astrophys. J., 257, 767.

Gallagher, J.S. and Starrfield, S.G. 1978. Ann. Rev. Astr. Astrophys., 16, 171.

Iben, I. Jr. 1982. Astrophys. J., 259, 244.

Iben, I. Jr. and Renzini, A. 1983. Ann. Rev. Astr. Astrophys., 21, 271.

Kenyon, S.J. and Truran, J.W. 1983. Astrophys. J., 273, 280.

Kenyon, S.J. and Webbink, R.F. 1984. Astrophys. J., 279, 252.

Kutter, G.S. and Sparks, W.M. 1980. Astrophys. J., 239, 988.

MacDonald, J. 1980. Mon. Not. Roy. Astr. Soc., 191, 933.

MacDonald, J. 1983. Astrophys. J., 267, 732.

Nariai, K., Nomoto, K., and Sugimoto, D. 1980. Pub. Astr. Soc. Japan, 32, 472.

Paczyński, B. 1970. Acta Astr., 21, 417.

Paczyński, B. and Rudak, B. 1980. Astr. Astrophys., 82, 349.

Paczynski, B. and Żytkow, A. 1978. Astrophys. J., 222, 604.

Prialnik, D. 1986. Astrophys. J., 310, 222.

Prialnik, D., Livio, M., Shaviv, G., and Kovetz, A. 1982. Astrophys. J., 257, 312.

Shustov, B.M., and Tutukov, A.V. 1985. Recent Results on Cataclysmic Variables (ESA SP-236), p. 113.

Sion, E.M., Acierno, M.J., and Tomczyk, S. 1979. Astrophys. J., 232, 832.

Sion, E.M., and Starrfield, S.G. 1985. in Recent Results on Cataclysmic Variables (ESA SP236), p. 17.

Starrfield, S., Sparks, W.M., and Truran, J.W. 1984, Astrophys. J., 291, 136.

Tutukov, A.V. and Yungel'son, L.R. 1976. Astrofizika, 12, n21.

Tutukov, A.V. and Yungel'son, L.R. 1982. in IAU Colloquium No. 70, The Nature of Symbiotic Stars, ed. M. Fricdjung and R. Viotti (Dordrecht: Reidel), p. 283.

Uus, U. 1970. Nauk Informatsii, 17, 32. 